\title{
Novel real-time PCR-based patho- and phylotyping of potentially zoonotic avian influenza A subtype H5 viruses at risk of incursion into Europe in 2017
}

\author{
MM Naguib ${ }^{12}$, A Graaf ${ }^{1}$, A Fortin ${ }^{3}$, C Luttermann ${ }^{4}$, U Wernery ${ }^{5}$, N Amarin ${ }^{6}$, HA Hussein ${ }^{7}$, H Sultan 8 , B Al Adhadh 9 , MK \\ Hassan ${ }^{2}$, M Beer $^{1}$, I Monne ${ }^{3}$, TC Harder ${ }^{1}$ \\ 1. Institute of Diagnostic Virology, Friedrich Loeffler Institute, Greifswald-Riems, Germany \\ 2. National Laboratory for Veterinary Quality Control on Poultry Production, Animal Health Research Institute, Giza, Egypt \\ 3. Istituto Zooprofilattico Sperimentale delle Venezie, Padua, Italy \\ 4. Institute of Immunology, Friedrich Loeffler Institute, Greifswald-Riems, Germany \\ 5. Central Veterinary Research Laboratory (CVRL), Dubai, United Arab Emirates \\ 6. Boehringer Ingelheim, Dubai, United Arab Emirates \\ 7. Faculty of Veterinary Medicine, Cairo University, Giza, Egypt \\ 8. Birds and Rabbits Medicine Department, Faculty of Veterinary Medicine, Sadat City University, Egypt \\ 9. Central Veterinary Laboratory, Ministry of Agriculture, Baghdad, Iraq
}

Correspondence: Timm Harder (timm.harder@fli.bund.de)

Citation style for this article:

Naguib MM, Graaf A, Fortin A, Luttermann C, Wernery U, Amarin N, Hussein HA, Sultan H, Al Adhadh B, Hassan MK, Beer M, Monne I, Harder TC. Novel realtime PCR-based patho- and phylotyping of potentially zoonotic avian influenza A subtype $\mathrm{H}_{5}$ viruses at risk of incursion into Europe in 2017 . Euro Surveill. 2017;22(1):pii=30435. DOI: http://dx.doi.org/10.2807/1560-7917.ES.2017.22.1.30435

Since November 2016, Europe witnesses another wave of incursion of highly pathogenic avian influenza (HPAl) $\mathrm{A}\left(\mathrm{H}_{5}\right)$ viruses of the Asian origin goose/ Guangdong (gs/GD) lineage. Infections with $\mathrm{H}_{5}$ viruses of clade 2.3.4.4b affect wild bird and poultry populations. $\mathrm{H}_{5}$ viruses of clades 2.2, 2.3.1.2C and 2.3.4.4a were detected previously in Europe in 2006, 2010 and 2014. Clades 2.2.1.2 and 2.3.2.1.C are endemic in Egypt and Western Africa, respectively and have caused human fatalities. Evidence exists of their cocirculation in the Middle East. Subtype $\mathrm{H}_{5}$ viruses of low pathogenicity (LPAI) are endemic in migratory wild bird populations. They potentially mutate into highly pathogenic phenotypes following transmission into poultry holdings. However, to date only the gs/ GD $\mathrm{H}_{5}$ lineage had an impact on human health. Rapid and specific diagnosis marks the cornerstone for control and eradication of HPAI virus incursions. We present the development and validation of five real-time RT-PCR assays (RT-qPCR) that allow sequencing-independent pathotype and clade-specific distinction of major gs/GD HPAI $\mathrm{H}_{5}$ virus clades and of Eurasian LPAI viruses currently circulating. Together with an influenza A virus-generic RT-qPCR, the assays significantly speed up time-to-diagnosis and reduce reaction times in a OneHealth approach of curbing the spread of gs/ GD HPAI viruses.

\section{Introduction}

Influenza A viruses constitute a virus species in the family Orthomyxoviridae. They harbour single-stranded negative-sense RNA arranged into eight genomic segments. Members of this species which infect avian hosts (avian influenza viruses, AIV) are grouped into $16\left(\mathrm{H}_{1}\right.$ to $\left.\mathrm{H}_{16}\right)$ and 9 ( $\mathrm{N}_{1}$ to $\left.\mathrm{N}_{9}\right)$ subtypes, respectively, based on phylogenetic and antigenic properties of their haemagglutinin ( $\mathrm{HA}$ ) and neuraminidase (NA) envelope glycoproteins [1]. Different species of aquatic wild birds are the natural reservoirs for all AIV subtypes. Novel subtypes and gene constellations continue to evolve in aquatic wild birds or in infected poultry populations by genetic reassortment during infection of a single host cell with two or more distinct AIV genotypes. In addition to reassortment, the intrinsically error-prone influenza virus genome replication machinery promotes the generation of quasi-species that can be shaped by directional selection pressures, e.g. following host species switches or by specific herd immunity. In the latter case, antigenic drift variants are selected that may escape immunity by very few amino acid substitutions in the HA [2].

Based on their virulence in galliform poultry (e.g. chicken, turkey), AIV are distinguished into groups of highly pathogenic (HP) and low pathogenic (LP) phenotypes [3]. Correct Al diagnosis includes determining the HA subtype and, in case of subtypes $\mathrm{H}_{5}$ or $\mathrm{H}_{7}$, also the pathotype. So far, HPAI phenotypes detected in the field (i.e. 'free' natural environment), were only described among AIV of subtypes $\mathrm{H}_{5}$ and $\mathrm{H}_{7}$ [4]. Some of these viruses including those of the HPAI $\mathrm{H}_{5}$ goose/ Guangdong (gs/GD) lineage that emerged in southern China in 1996, have zoonotic potential and are sporadically transmitted from infected birds to humans $[5,6]$. HPAl viruses of the gs/GD lineage have continued to circulate and evolved into numerous clades. Viruses 


\section{FIGURE 1}

Evaluation of detection limits and precision of pathotyping and phylotyping quantitative reverse transcription PCRs compared with a generic matrix $(\mathrm{M})$ gene RT-qPCR
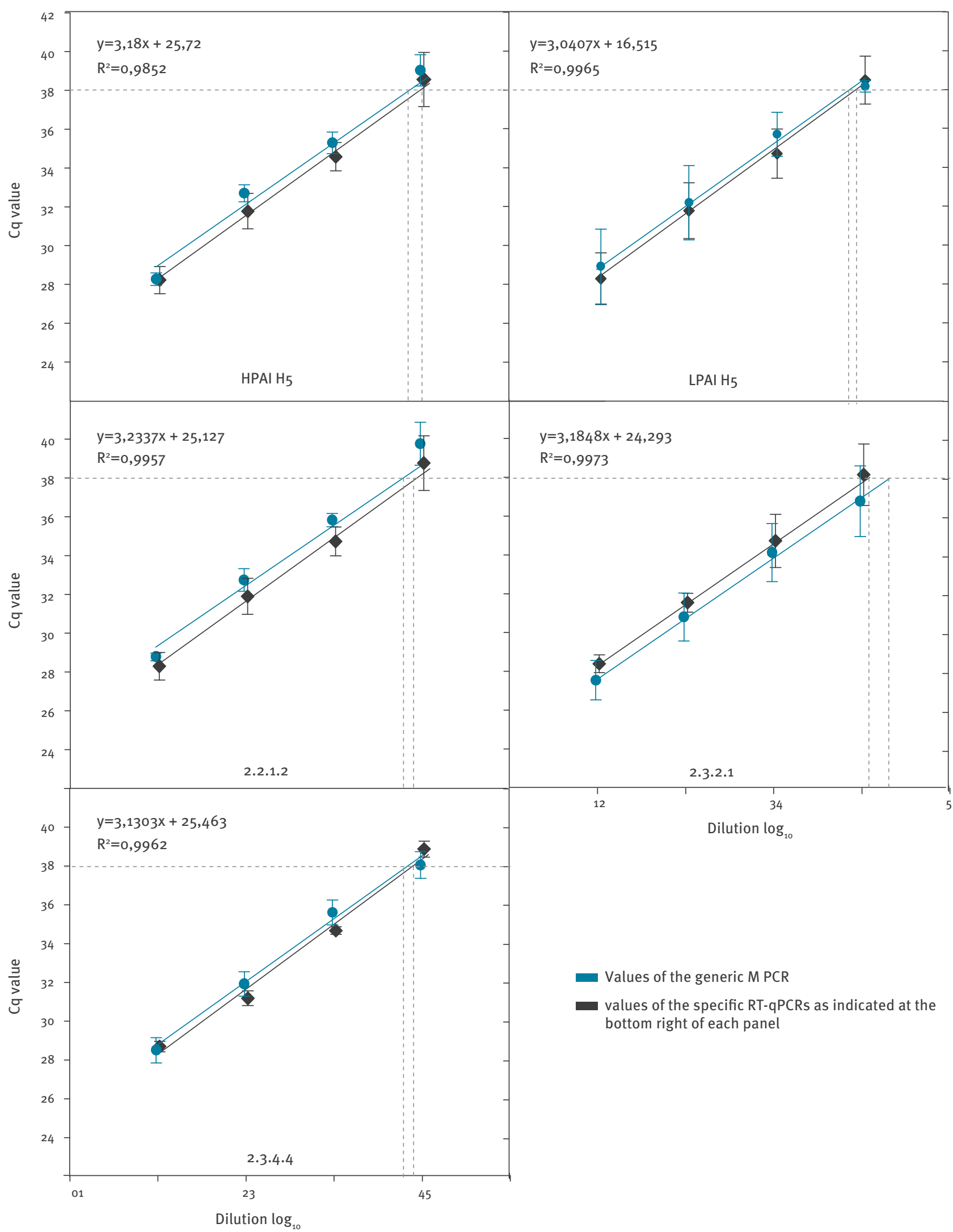

Values of the generic M PCR

values of the specific RT-qPCRs as indicated at the bottom right of each panel

Cq: cycle of quantification; HPAl: highly pathogenic avian influenza; LPAI: low pathogenic avian influenza; M: matrix; RT-qPCR: quantitative reverse transcription PCR.

The detection limit was determined based on triplicate analyses of serial 10-fold dilutions of target RNA of reference viruses: HPAI H5: A/duck/Egypt/AR236-A3NLQP/2015 ( $\left.\mathrm{H}_{5} \mathrm{~N}_{1}\right)$; LPAI H5: A/teal-Foehr/Wv1378-79/2003 ( $\left.\mathrm{H}_{5} \mathrm{~N}_{2}\right)$ (upper panel); HPAl H5 clade 2.2.1.2: A/duck/Egypt/AR236-A3NLQP/2015 (H5N1); clade 2.3.2.1: A/quail/Dubai/ AR3445-2504.3/2014 ( $\left.\mathrm{H}_{5} \mathrm{~N}_{1}\right)$ and clade 2.3.4.4: A/turkey/Germany-MV/R2472/2014 ( $\left.\mathrm{H}_{5} \mathrm{~N} 8\right)$ (lower panel). A cut-off value of $\mathrm{Cq} 38$ was chosen to calculate limits of detection and

a Described in [29]. 
Pathotyping and phylotyping of virus isolates and clinical samples of potentially zoonotic Eurasian avian influenza A subtype $\mathrm{H} 5$ viruses by quantitative reverse transcription PCRs
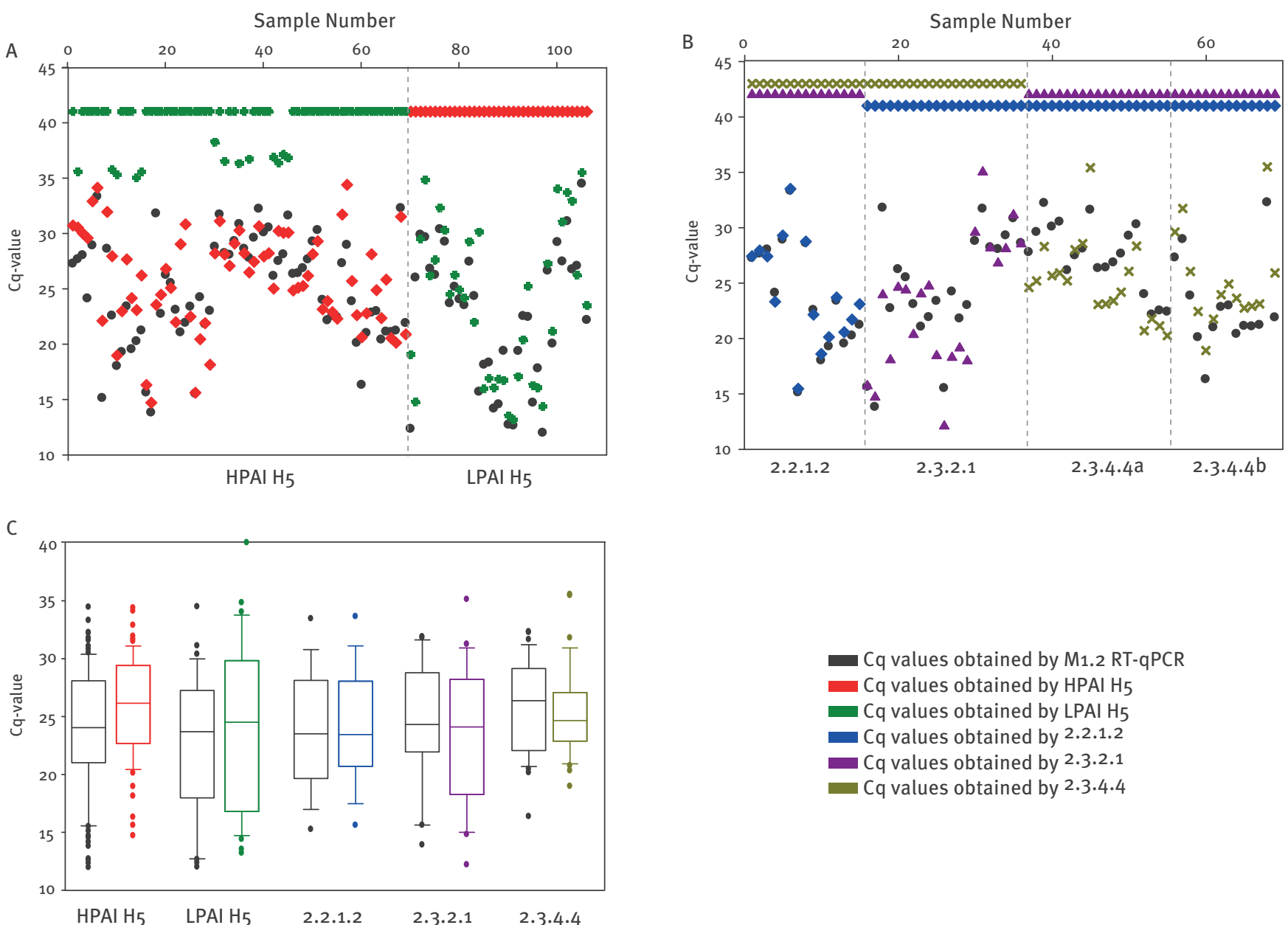

Cq: cycle of quantification; HPAI: highly pathogenic avian influenza; LPAI: low pathogenic avian influenza; M: matrix; RT-qPCR: quantitative reverse transcription $P C R$.

Sample numbers in A and B refer to Table 3. Cq values obtained for each sample by M1.2 RT-qPCR are shown as black dots (panels A and B); Cq values obtained for each sample by the specific RT-qPCRs are depicted as follows: Panel A red lozenges - HPAI H5, green crosses - LPAl $\mathrm{H}_{5}$; panel B blue lozenges - clade 2.2.1.2, purple triangles - clade 2.3.2.1, ochre Xs - clade 2.3.4.4.; panel C compares categorised Cq values obtained for all samples by M1.2 RT-qPCR (black box-and-whiskers) and the specific RT-qPCRs (colours as described for panels A and B).

of three major phylogenetic clades (2.2.1.2, 2.3.2.1 and 2.3 .4 .4$)$ as well as of three further minor clades (1.1.2, 2.1.3.2 and 7.2) have become endemic in poultry populations in several countries in Asia, Africa and the Middle East [7]. Occasionally, spillover transmission from infected poultry may cause infection and viral spread in wild birds with increased mortality in some species. Infected migratory wild birds may spread such viruses across wider distances and act as the source of transmission back to poultry $[7,8]$.

Europe has experienced several incursions by viruses of the gs/GD lineage over the past decade; both wild birds and poultry were affected but no human cases were reported [9]. This is in sharp contrast to Egypt and Asian countries where the endemicity of HPAI $\mathrm{H}_{5}$ viruses in poultry is associated with repeated spillover transmission to and infection of humans. In fact, the majority of human $\mathrm{HPAl} \mathrm{H}_{5}$ cases worldwide were registered in Egypt $[10,11]$. Moreover, a new major clade, designated 2.2.1.2, evolved along with transient spread of an escape mutant-based lineage, 2.2.1.1, in this country [12].

Further potentially zoonotic gs/GD viruses of clade 2.3.2.1C are widespread in Central and Southern Asia and they were sporadically detected along the European Black Sea coast as well as in the Middle East [13-15]. In addition, viruses of this clade have caused major outbreaks among poultry in several Western African countries with ongoing virus circulation to date [16]. Interestingly, 2.3.2.1C viruses have not (yet) been reported from Egypt. Since 2010, another gs/GD cluster, termed 2.3.4.4, has evolved in eastern China 
TABLE 1

Primers and probes designed for differentiating pathotype and phylotype of Eurasian wild bird and goose/Guangdong origin potentially zoonotic avian influenza A subtype H5 viruses

\begin{tabular}{|c|c|c|c|c|c|}
\hline Primer/Probe ID & Target & Sequence ( $5^{\prime}$ to $\left.3^{\prime}\right)$ & Location & $\begin{array}{l}\text { Amplicon } \\
\text { size }\end{array}$ & $\begin{array}{c}\text { Accession } \\
\text { number }\end{array}$ \\
\hline $\mathrm{H}_{5}$ HP_EA_F1 & \multirow{4}{*}{ HPAI $\mathrm{H}_{5}$} & CCTTGCDACTGGRCTCAG & $984-1001$ & \multirow{4}{*}{109} & \multirow{4}{*}{ EPI647540 } \\
\hline $\mathrm{H}_{5}$ HP_EA_F2 & & TCCTTGCAACAGGACTAAG & $983-1001$ & & \\
\hline H5_HP_EA_probe & & FAM- AAGAARAAARAGAGGACTRTTTGGAGCT-BHQ-1 & $1023-1050$ & & \\
\hline H5_HP_EA_R & & GTCTACCATTCCYTGCCA & $1092-1075$ & & \\
\hline $\mathrm{H}_{5} \mathrm{LP}-\mathrm{EA} \_\mathrm{F}$ & \multirow{3}{*}{ LPAI $\mathrm{H}_{5}$} & CCCAAATACGTGAAATCAGAT & $955-975$ & \multirow{3}{*}{133} & \multirow{3}{*}{$\mathrm{EPI} 356413$} \\
\hline $\mathrm{H}_{5} \mathrm{LP}_{1}$ EA_probe & & FAM-CCAAATAGYCCTCTYGTYTCT-BHQ-1 & $1052-1072$ & & \\
\hline $\mathrm{H}_{5} \mathrm{LP}-\mathrm{EA} \_\mathrm{R}$ & & GCC ACC CTC CTT CTA TAA AG & $1088-1069$ & & \\
\hline $\mathrm{H}_{5} \_$2.2.1.2_Fw & \multirow{3}{*}{ Clade 2.2.1.2 } & CATTTTGAGAAAATTCAGATCATT & $376-399$ & \multirow{3}{*}{161} & \multirow{3}{*}{ EPI573250 } \\
\hline $\mathrm{H}_{5} \_2.2 .1 .2 \_$probe & & FAM-TCCATACCARGGAAGATCCTCCTTT-BHQ-1 & $451-474$ & & \\
\hline $\mathrm{H}_{5} \_2.2 .1 .2 \_\mathrm{Rev}$ & & GGTATGCATCGTTCTTTTTGG & $537-517$ & & \\
\hline $\mathrm{H}_{5 \_}$2.3.2.1_F & \multirow{3}{*}{ Clade 2.3.2.1 } & GAGATTGGTACCAAAAATAGCC & $669-690$ & \multirow{3}{*}{146} & \multirow{3}{*}{ EPI603577 } \\
\hline $\mathrm{H}_{5 \_2}$.3.2.1_probe & & FAM-ACGGGCAAAGTGGCAGGATAGATTTC-BHQ-1 & $707-732$ & & \\
\hline $\mathrm{H}_{5} \_2.3 \cdot 2.1 \_\mathrm{R}$ & & CAATGAAATTTCCATTACTCTCG & $815-793$ & & \\
\hline $\mathrm{H}_{5} \_2 \cdot 3 \cdot 4 \cdot 4 \_\mathrm{F} \_\mathrm{A}$ & \multirow{4}{*}{$\begin{array}{c}\text { Clade } \\
2 \cdot 3 \cdot 4 \cdot 4\end{array}$} & ATACCAGGGAGCATCCTCA & $484-502$ & \multirow{5}{*}{114} & \multirow{4}{*}{ EPI554605 } \\
\hline $\mathrm{H}_{5} \_2 \cdot 3 \cdot 4 \cdot 4 \_\mathrm{F} \_\mathrm{B}$ & & ATACCAGGGAACGCCCTCC & $484-502$ & & \\
\hline H5_2.3.4.4_probe & & FAM-TCGTTCTTTTTGATGAGCCATACCACA-BHQ-1 & $540-560$ & & \\
\hline $\mathrm{H}_{5} \_2 \cdot 3 \cdot 4 \cdot 4 \_\mathrm{R} \_\mathrm{A}$ & & ATTATTGTAGCTTATCTTTATTGTC & $598-574$ & & \\
\hline $\mathrm{H}_{5} \_2 \cdot 3 \cdot 4 \cdot 4 \_\mathrm{R} \_\mathrm{B}$ & & ATTATTGTAGCTTATCTTTATTGTT & $598-574$ & & \\
\hline
\end{tabular}

gs/GD: goose/Guangdong; HA: haemagglutinin; ID: identity.

a Accession number used to describe the position of the oligonucleotide along the HA gene. Sequences were obtained from GenBank at the National Center for Biotechnology Information (NCBI) or the EpiFlu database of the Global Initiative on Sharing Avian Influenza Data (GISAID).

and on the Korean peninsula [17]. These viruses have revealed a strong propensity to reassort with other influenza subtypes giving rise to novel HPAl sub- and genotypes including influenza $A\left(\mathrm{H}_{5} \mathrm{N6}\right)$ and $A\left(\mathrm{H}_{5} \mathrm{~N} 8\right)$. The latter subtype has proven to be highly mobile and was carried by infected wild birds to Europe and the North American continent in late $2014[8,18]$. In November 2016, HPAI H5N8 viruses of the 2.3.4.4 clade re-emerged on a large scale in wild birds in several central European countries and caused considerable mortality especially among diving duck species; sporadic incursions into poultry holdings were documented as well [19]. At the same time, this lineage was also detected in poultry in Israel [20].

Eurasian-origin LPAI subtype $\mathrm{H}_{5}$ viruses distantly related to the gs/GD lineage are routinely detected in aquatic wild bird populations with peak incidences during the autumn migration period [21]. Spillover of LPAI virus into poultry may cause notifiable outbreaks and bears the risk of the de novo generation of HP phenotypes following spontaneous mutations [3]. No human LPAI $\mathrm{H}_{5}$ virus infections have been reported so far.
Continuous co-circulation in poultry and sporadic spillover into migratory wild bird populations of different endemic $\mathrm{HPAl}_{5}$ virus lineages poses constant risks of new incursions into Europe by migrating wild birds or in association with (illegal) poultry trading practices [9]. Furthermore, co-circulation of various HPAl lineages with different antigenic properties potentiates problems of control and eradication. Given the zoonotic propensities of some of the $\mathrm{H}_{5}$ viruses, tight control of infections in poultry is essential to curtail risks of human infections and further spread $[22,23]$. Molecular diagnosis including patho- and phylotyping of the relevant AIV is an important prerequisite for effective control measures.

We developed rapid diagnostic solutions on the basis of quantitative reverse transcription real-time $P C R$ assays (RT-qPCR), to pathotype, without sequencing, gs/GD lineage HPAI and Eurasian LPAI $\mathrm{H}_{5}$ subtype viruses, and to distinguish HPAl gs/GD viruses of clades 2.2.1.2, 2.3.2.1 and 2.3.4.4, including viruses of the ongoing 2016 epizootic in Europe. 
TABLE 2

Reference viruses used to determine analytical specificity of five PCR assays to detect potentially zoonotic avian influenza subtype $\mathrm{H} 5$ viruses

\begin{tabular}{|c|c|c|c|c|c|c|c|c|}
\hline \multirow{2}{*}{\multicolumn{2}{|c|}{ Reference virus }} & \multirow{3}{*}{$\begin{array}{c}\text { Accession } \\
\text { number of } \mathrm{HA}^{\mathrm{a}}\end{array}$} & \multirow{3}{*}{$\begin{array}{l}\begin{array}{l}\text { Patho-and } \\
\text { Phylotype }\end{array} \\
\text { HP Clade } 2.2\end{array}$} & \multicolumn{5}{|c|}{ PCR method ${ }^{b}$} \\
\hline & & & & \multirow{2}{*}{$\begin{array}{c}\text { HPAI H5 } \\
\text { Pos }\end{array}$} & \multirow{2}{*}{$\begin{array}{c}\text { LPAI H5 } \\
\text { Neg }\end{array}$} & \multirow{2}{*}{$\begin{array}{c}\text { Clade } \\
2.2 .1 .2 \\
\text { Pos } \\
\end{array}$} & \multirow{2}{*}{\begin{tabular}{c|} 
Clade \\
$2.3 \cdot 2.1$ \\
$\mathbf{N e g}$ \\
\end{tabular}} & \multirow{2}{*}{$\begin{array}{c}\text { Clade } \\
2.3 \cdot 4 \cdot 4 \\
\text { Neg }\end{array}$} \\
\hline 1 & $\mathrm{~A} /$ turkey/Turkey/1/2005 $\left(\mathrm{H}_{5} \mathrm{~N}_{1}\right)$ & & & & & & & \\
\hline 2 & $\begin{array}{c}\text { A/chicken/Egypt/o879-NLQP/R737/2008 } \\
\left(\mathrm{H}_{5} \mathrm{~N}_{1}\right)\end{array}$ & GQ184238 & $\begin{array}{l}\text { HP Clade } \\
2.2 .1 .1\end{array}$ & Pos & Neg & Neg & Neg & Neg \\
\hline 3 & $\begin{array}{c}\text { A/chicken/Egypt/NLQP7FL-AR747/ } 2013 \\
\left(\mathrm{H}_{5} \mathrm{~N}_{1}\right)\end{array}$ & $\mathrm{EPI}_{557170}$ & $\begin{array}{l}\text { HP Clade } \\
2.2 .1 .2\end{array}$ & Pos & Neg & Pos & Neg & Neg \\
\hline 4 & $\begin{array}{c}\text { A/duck/Egypt/AR236-A3NLQP/2015 } \\
\left(\mathrm{H}_{5} \mathrm{N1}_{1}\right)\end{array}$ & EPI573260 & $\begin{array}{l}\text { HP Clade } \\
2.2 .1 .2\end{array}$ & Pos & Neg & Pos & Neg & Neg \\
\hline 5 & $\begin{array}{c}\text { A/turkey/Egypt/AR238-SD177NLQP/2014 } \\
\left(\mathrm{H}_{5} \mathrm{~N}_{1}\right)\end{array}$ & EPI573268 & $\begin{array}{l}\text { HP Clade } \\
2.2 .1 .2\end{array}$ & Pos & Neg & Pos & Neg & Neg \\
\hline 6 & $\begin{array}{c}\text { A/peregrine falcon/Dubai/AR3430/2014 } \\
\left(\mathrm{H}_{5} \mathrm{~N}_{1}\right)\end{array}$ & EPI603553 & $\begin{array}{l}\text { HP Clade } \\
2.3 .2 .1 \mathrm{C}\end{array}$ & Pos & Neg & Neg & Pos & Neg \\
\hline 7 & $\begin{array}{c}\text { A/quail/Dubai/AR3445-2504.3/2014 } \\
\text { (H5N1) }\end{array}$ & EPI603577 & $\begin{array}{l}\text { HP Clade } \\
2.3 \cdot 2.1 \mathrm{C}\end{array}$ & Pos & Neg & Neg & Pos & Neg \\
\hline 8 & $\begin{array}{c}\text { A/duck/Bangladesh/D3-AR2111/2013 } \\
\left(\mathrm{H}_{5} \mathrm{~N}_{1}\right)\end{array}$ & $\mathrm{SA}^{c}$ & $\begin{array}{l}\text { HP Clade } \\
2.3 .2 .1 \mathrm{a}\end{array}$ & Pos & Neg & Neg & Pos & Neg \\
\hline 9 & $\begin{array}{c}\text { A/turkey/Germany/AR2485-86/2014 } \\
\left(\mathrm{H}_{5} \mathrm{~N} 8\right)\end{array}$ & $\mathrm{EPI} 552746$ & $\begin{array}{l}\text { HP Clade } \\
2 \cdot 3 \cdot 4 \cdot 4 \mathrm{a}\end{array}$ & Pos & Neg & Neg & Neg & Pos \\
\hline 10 & $\begin{array}{c}\text { A/turkey/Germany-MV/AR2472/2014 } \\
\left(\mathrm{H}_{5} \mathrm{~N} 8\right)\end{array}$ & $\mathrm{EPI}_{544756}$ & $\begin{array}{l}\text { HP Clade } \\
2.3 \cdot 4 \cdot 4 \mathrm{a}\end{array}$ & Pos & Neg & Neg & Neg & Pos \\
\hline 11 & $\begin{array}{c}\text { A/tufted duck/Germany/AR8444/2016 } \\
\left(\mathrm{H}_{5} \mathrm{~N} 8\right)\end{array}$ & EPI859212 & $\begin{array}{l}\text { HP Clade } \\
2 \cdot 3 \cdot 4 \cdot 4 \mathrm{~b}\end{array}$ & Pos & Neg & Neg & Neg & Pos \\
\hline 12 & A/chicken/Indonesia/R132/2004 ( $\left.\mathrm{H}_{5} \mathrm{~N}_{1}\right)$ & $\mathrm{EPI}_{354072}$ & $\begin{array}{c}\text { HP Clade } \\
2.1 .1 \\
\end{array}$ & Pos & Neg & Neg & Neg & Neg \\
\hline 13 & A/chicken/Indonesia/R134/2003 ( $\left.{ }_{5} \mathrm{~N}_{1}\right)$ & AM183669 & $\begin{array}{c}\text { HP Clade } \\
2.1 .1 \\
\end{array}$ & Pos & Neg & Neg & Neg & Neg \\
\hline 14 & $\mathrm{~A} /$ chicken/Indonesia/R60/2005 ( $\left.\mathrm{H}_{5} \mathrm{~N}_{1}\right)$ & AM183670 & $\begin{array}{l}\text { HP Clade } \\
2.1 .1\end{array}$ & Pos & Neg & Neg & Neg & Neg \\
\hline 15 & A/Vietnam/1194/2004 ( $\left.\mathrm{H}_{5} \mathrm{~N}_{1}\right)$ & GQ149236 & HP Clade 1.1 & Pos & Neg & Neg & Neg & Neg \\
\hline 16 & $\mathrm{~A} /$ chicken/GXLA/1204/2004 ( $\left.\mathrm{H}_{5} \mathrm{~N}_{1}\right)$ & AM183671 & HP Clade 2.4 & Pos & Neg & Neg & Neg & Neg \\
\hline 17 & A/chicken/Vietnam/P41/2005 ( $\left.\mathrm{H}_{5} \mathrm{~N}_{1}\right)$ & AM183672 & HP Clade 1.1 & Pos & Neg & Neg & Neg & Neg \\
\hline 18 & A/chicken/Vietnam/P78/2005 ( $\left.\mathrm{H}_{5} \mathrm{~N}_{1}\right)$ & AM183673 & HP Clade 1.1 & Pos & Neg & Neg & Neg & Neg \\
\hline 19 & $\begin{array}{c}\text { A/common teal/Germany/Wv1378- } \\
79 / 2003\left(\mathrm{H}_{5} \mathrm{~N} 2\right)\end{array}$ & $\mathrm{HF}_{5} 63058$ & LP & Neg & Pos & Neg & Neg & Neg \\
\hline 20 & A/duck/Germany/R1789/2008 ( $\left.\mathrm{H}_{5} \mathrm{~N}_{3}\right)$ & CY107849 & LP & Neg & Pos & Neg & Neg & Neg \\
\hline 21 & A/turkey/Germany/AR915/2015 (H7N7) & $\mathrm{SA}^{\mathrm{c}}$ & $\mathrm{H}_{7} \mathrm{~N}_{7}$ & Neg & Neg & Neg & Neg & Neg \\
\hline 22 & A/chicken/Egypt/AR754-14/2013 (H9N2) & $\mathrm{EPI}_{557457}$ & $\mathrm{H} 9 \mathrm{~N}_{2}$ & Neg & Neg & Neg & Neg & Neg \\
\hline 23 & A/chicken/Sudan/AR251-15/2014 (IBV) & $\mathrm{KX} 272465$ & IBV & Neg & Neg & Neg & Neg & Neg \\
\hline 24 & A/chicken/Egypt/AR254-15/2014 (NDV) & $\mathrm{SA}^{\mathrm{c}}$ & NDV & Neg & Neg & Neg & Neg & Neg \\
\hline
\end{tabular}

Cq: cycle of quantification; HA: haemagglutinin; HP: highly pathogenic; HPAI: highly pathogenic avian influenza; IBV: infectious bronchitis virus; LP: low pathogenic; LPAI: low pathogenic avian influenza; NDV: Newcastle disease virus; Neg: negative; Pos: positive; RT-qPCR: quantitative reverse transcription PCR; SA: sequences available.

a Sequences were obtained from GenBank at the National Center for Biotechnology Information (NCBI) or the EpiFlu database of the Global Initiative on Sharing Avian Influenza Data (GISAID).

b Positive results: Cq value in similar range as with influenza A virus generic M RT-qPCR; negative results: Cq>40. ${ }^{c}$ Sequenced in the frame of the current study; sequences available from the authors upon request.

\section{Methods}

\section{Virus isolates and clinical samples}

A total of 24 reference virus isolates were obtained from the virus repositories at the Friedrich Loeffler Institute, Greifswald-Riems, Germany, or were provided by the National Laboratory for quality control on poultry production in Giza, Egypt, and by the Central
Veterinary Research Laboratory (CVRL) in Dubai, United Arab Emirates (see also first table under Results).

Moreover, 106 field samples were included. These were obtained from holdings of different poultry sectors and wild birds from countries in Western Europe (Germany), the Middle East (Egypt, Iraq, United Arab Emirates) and Western Africa (Burkina Faso, Cameroon, Ghana, Ivory Coast, Niger), for HPAI viruses in the period between 
TABLE 3A

Pathotyping and phylotyping of different potentially zoonotic HPAI and LPAI influenza A subtype H5 virus isolates and field samples collected from poultry and wild bird species in different countries, 2013-2016

\begin{tabular}{|c|c|c|c|c|c|c|c|c|c|c|}
\hline \multirow{2}{*}{ No. } & \multirow{2}{*}{ Sample ID } & \multirow{2}{*}{ Type of sample } & \multirow{2}{*}{$\begin{array}{l}\text { Accession } \\
\text { Number }{ }^{a}\end{array}$} & \multirow{2}{*}{ Clade } & \multicolumn{6}{|c|}{ PCR results } \\
\hline & & & & & M1.2 & HPAI $\mathrm{H}_{5}$ & LPAI $\mathrm{H}_{5}$ & 2.2 .1 .2 & $2 \cdot 3 \cdot 2.1$ & $2.3 \cdot 4 \cdot 4$ \\
\hline 1 & A/chicken/Egypt/NLQP33SD-AR748/2013 & Isolate & $\mathrm{EPI} 557178$ & HP 2.2.1.2 & 27.25 & 30.72 & Neg & 27.43 & Neg & $\mathrm{Neg}$ \\
\hline 2 & A/chicken/Egypt/NLQP2AL-AR749/2013 & Isolate & $\mathrm{EPI}_{557186}$ & HP 2.2.1.2 & 27.65 & 30.53 & 35.56 & 27.94 & Neg & Neg \\
\hline 3 & A/duck/Egypt/NLQP27SG-AR750/2013 & Isolate & EPI557194 & HP 2.2.1.2 & 28.01 & 30.01 & Neg & 27.41 & Neg & Neg \\
\hline 4 & A/chicken/Egypt/NLQP639V-AR752/2013 & Isolate & EPI557202 & HP 2.2.1.2 & 24.11 & 29.58 & Neg & 23.32 & Neg & Neg \\
\hline 5 & A/chicken/Egypt/NLQP20SL-AR751/2013 & Isolate & EPI557210 & HP 2.2.1.2 & 28.90 & 32.90 & Neg & 29.30 & Neg & Neg \\
\hline 6 & A/chicken/Egypt/NLQP139V-AR753/2013 & Isolate & $\mathrm{EPI} 557218$ & HP 2.2.1.2 & 33.32 & 34.13 & Neg & 33.51 & Neg & Neg \\
\hline 7 & A/quail/Egypt/BSU5514-AR2219/2014 & Field sample & $\mathrm{EPI}_{557138}$ & HP 2.2.1.2 & 15.12 & 22.12 & $\mathrm{Neg}$ & 15.47 & Neg & Neg \\
\hline 8 & A/chicken/Egypt/AR234-FAOF8NLQP/2014 & Field sample & EPI573250 & HP 2.2.1.2 & 28.60 & 31.95 & Neg & 28.75 & Neg & Neg \\
\hline 9 & A/turkey/Egypt/AR235-S240NLQP/2014 & Field sample & $\mathrm{EPI} 573252$ & HP 2.2.1.2 & 22.56 & 27.94 & 35.77 & 22.16 & Neg & Neg \\
\hline 10 & A/chicken/Egypt/AR3690A/2016 & Field sample & $\mathrm{SA}^{\mathrm{b}}$ & HP 2.2.1.2 & 18.01 & 18.97 & 35.29 & 18.61 & $\mathrm{Neg}$ & Neg \\
\hline 11 & A/chicken/Egypt/AR3706/2016 & Field sample & $\mathrm{SA}^{\mathrm{b}}$ & HP 2.2.1.2 & 19.27 & 22.98 & Neg & 20.13 & Neg & Neg \\
\hline 12 & A/chicken/Egypt/AR3707/2016 & Field sample & $\mathrm{SA}^{\mathrm{b}}$ & HP 2.2.1.2 & 23.39 & 27.66 & $\mathrm{Neg}$ & 23.71 & Neg & Neg \\
\hline 13 & A/chicken/Egypt/AR3737/2016 & Field sample & $\mathrm{SA}^{\mathrm{b}}$ & HP 2.2.1.2 & 19.53 & 24.16 & Neg & 20.58 & $\mathrm{Neg}$ & Neg \\
\hline 14 & A/chicken/Egypt/AR3741/2016 & Field sample & $\mathrm{SA}^{\mathrm{b}}$ & HP 2.2.1.2 & 20.25 & 23.08 & 35.04 & 21.71 & Neg & Neg \\
\hline 15 & A/chicken/Egypt/AR3753/2016 & Field sample & $\mathrm{SA}^{\mathrm{b}}$ & HP 2.2.1.2 & 21.22 & 26.21 & 35.55 & 23.10 & Neg & Neg \\
\hline 16 & A/seagull/Dubai/AR3443-2504.1/2014 & Isolate & EPI603554 & HP 2.3.2.1 & 15.62 & 16.32 & Neg & Neg & 15.72 & Neg \\
\hline 17 & A/stone curlew/Dubai/AR3444-2504.2/2014 & Isolate & EPI603569 & HP 2.3.2.1 & 13.81 & 14.72 & Neg & Neg & 14.70 & Neg \\
\hline 18 & A/duck/Ivory_Coast/15VIR2742-1/2015 & Spleen and caecum & NA & HP 2.3.2.1 & 31.79 & 23.56 & Neg & Neg & 23.93 & Neg \\
\hline 19 & A/chicken/Ghana/15VIR2588-4/2015 & Spleen & KU97137 & HP 2.3.2.1 & 22.72 & 24.47 & Neg & Neg & 18.07 & Neg \\
\hline 20 & A/chicken/Ghana/15VIR2588-10/2015 & Cloacal swab & KU971357 & HP 2.3.2.1 & 26.24 & 26.80 & $\mathrm{Neg}$ & Neg & 24.61 & Neg \\
\hline 21 & $\mathrm{~A} /$ chicken/Niger/15VIR2060-12/2015 & Tracheal swab & KU971309 & HP 2.3.2.1 & 25.50 & 25.08 & Neg & Neg & 24.37 & Neg \\
\hline 22 & A/chicken/Niger/15VIR2060-5/2015 & Swab & KU971326 & HP 2.3.2.1 & 23.08 & 21.99 & Neg & Neg & 20.35 & Neg \\
\hline 23 & A/domestic_bird/Burkina_Faso/15VIR1774-24/2015 & Swab & KU971508 & HP 2.3.2.1 & 21.05 & 29.03 & Neg & Neg & 24.01 & Neg \\
\hline 24 & A/domestic_bird/Burkina_Faso/15VIR1774-23/2015 & Organ & KU971500 & HP 2.3.2.1 & 21.91 & 30.83 & Neg & Neg & 24.72 & Neg \\
\hline 25 & A/chicken/Ghana/16VIR-4304-1/2016 & Organ & $\mathrm{SA}^{\mathrm{b}}$ & HP 2.3.2.1 & 23.37 & 22.49 & Neg & Neg & 18.44 & Neg \\
\hline 26 & A/chicken/Ghana/16VIR-4304-25/2016 & Organ & $\mathrm{SA}^{\mathrm{b}}$ & HP 2.3.2.1 & 15.51 & 15.62 & Neg & Neg & 12.09 & Neg \\
\hline 27 & A/chicken/Ghana/16VIR-4304-42/2016 & Organ & $\mathrm{SA}^{\mathrm{b}}$ & HP 2.3.2.1 & 24.22 & 20.45 & Neg & Neg & 18.28 & Neg \\
\hline 28 & A/chicken/Ghana/16VIR-4304-9/2016 & Organ & $\mathrm{SA}^{\mathrm{b}}$ & HP 2.3.2.1 & 21.79 & 21.90 & $\mathrm{Neg}$ & Neg & 19.13 & Neg \\
\hline 29 & A/duck/Cameroon/16VIR-3791-21/2016 & Lung and trachea & $\mathrm{SA}^{\mathrm{b}}$ & HP 2.3.2.1 & 23.00 & 18.14 & Neg & Neg & 17.98 & Neg \\
\hline 30 & A/chicken/Iraq/AR5282/2016 & Field sample & NA & HP 2.3.2.1 & 28.78 & 28.20 & Neg & Neg & 29.57 & Neg \\
\hline 31 & A/chicken/Iraq/AR5283/2016 & Field sample & NA & HP 2.3.2.1 & 31.70 & 31.12 & Neg & Neg & 35.02 & Neg \\
\hline 32 & A/chicken/Iraq/AR5286/2016 & Field sample & $\mathrm{SA}^{\mathrm{b}}$ & HP 2.3.2.1 & 28.21 & 28.10 & 36.50 & Neg & 28.16 & Neg \\
\hline 33 & A/chicken/Iraq/AR5287/2016 & Field sample & $\mathrm{SA}^{\mathrm{b}}$ & HP 2.3.2.1 & 28.05 & 27.08 & Neg & Neg & 26.80 & Neg \\
\hline 34 & A/chicken/Iraq/AR5291/2016 & Field sample & $\mathrm{SA}^{\mathrm{b}}$ & HP 2.3.2.1 & 29.29 & 29.09 & $\mathrm{Neg}$ & Neg & 28.09 & Neg \\
\hline 35 & A/chicken/Iraq/AR5292/2016 & Field sample & NA & HP 2.3.2.1 & 30.83 & 30.28 & 36.32 & $\mathrm{Neg}$ & 31.15 & $\mathrm{Neg}$ \\
\hline 36 & A/chicken/Iraq/AR5296/2016 & Field sample & $\mathrm{SA}^{\mathrm{b}}$ & HP 2.3.2.1 & 28.60 & 28.21 & Neg & Neg & 28.53 & Neg \\
\hline 37 & A/turkey/Germany/AR2499/2014 & Field sample & $\mathrm{SA}^{\mathrm{b}}$ & HP 2.3.4.4 & 27.78 & 26.48 & 36.71 & Neg & Neg & 24.61 \\
\hline 38 & A/turkey/Germany/AR2500/2014 & Field sample & $\mathrm{SA}^{\mathrm{b}}$ & HP 2.3.4.4 & 29.59 & 27.44 & Neg & Neg & Neg & 25.20 \\
\hline 39 & A/turkey/Germany/AR2501/2014 & Field sample & $\mathrm{SA}^{\mathrm{b}}$ & HP 2.3.4.4 & 32.21 & 30.65 & $\mathrm{Neg}$ & Neg & Neg & 28.30 \\
\hline 40 & A/turkey/Germany/AR2502/2014 & Field sample & $\mathrm{SA}^{\mathrm{b}}$ & HP 2.3.4.4 & 30.08 & 27.92 & Neg & Neg & Neg & 25.67 \\
\hline 41 & A/turkey/Germany/AR2503/2014 & Field sample & $\mathrm{SA}^{\mathrm{b}}$ & HP 2.3.4.4 & 30.52 & 28.21 & Neg & Neg & Neg & 25.92 \\
\hline 42 & A/turkey/Germany/AR2562/2014 & Field sample & $\mathrm{SA}^{\mathrm{b}}$ & HP 2.3.4.4 & 26.15 & 25.02 & 36.88 & Neg & Neg & 25.21 \\
\hline 43 & A/turkey/Germany/AR2574/2014 & Field sample & $\mathrm{SA}^{\mathrm{b}}$ & HP 2.3.4.4 & 27.49 & 30.23 & 36.36 & Neg & $\mathrm{Neg}$ & 28.01 \\
\hline 44 & A/turkey/Germany/AR2591/2014 & Field sample & $\mathrm{SA}^{\mathrm{b}}$ & HP 2.3.4.4 & 28.09 & 30.06 & 37.13 & Neg & Neg & 28.57 \\
\hline 45 & A/teal/Germany/AR2917/2014 & Field sample & $\mathrm{SA}^{\mathrm{b}}$ & HP 2.3.4.4 & 31.60 & 30.08 & 36.82 & Neg & Neg & 35.41 \\
\hline 46 & A/turkey/Germany/AR3372/2014 & Field sample & $\mathrm{EPI}_{553172}$ & HP 2.3.4.4 & 26.33 & 24.85 & Neg & Neg & Neg & 23.07 \\
\hline 47 & A/turkey/Germany/AR3376/2014 & Field sample & $\mathrm{SA}^{\mathrm{b}}$ & HP 2.3.4.4 & 26.39 & 25.10 & Neg & Neg & Neg & 23.12 \\
\hline 48 & A/turkey/Germany/AR3381/2014 & Field sample & $\mathrm{SA}^{\mathrm{b}}$ & HP 2.3.4.4 & 26.85 & 25.26 & Neg & Neg & Neg & 23.40 \\
\hline 49 & A/turkey/Germany/AR3382/2014 & Field sample & $\mathrm{SA}^{\mathrm{b}}$ & HP 2.3.4.4 & 27.64 & 26.18 & $\mathrm{Neg}$ & $\mathrm{Neg}$ & Neg & 24.18 \\
\hline 50 & A/turkey/Germany/AR3383/2014 & Field sample & $S A^{b}$ & HP 2.3.4.4 & 29.26 & 28.13 & Neg & Neg & Neg & 26.06 \\
\hline 51 & A/duck/Germany/AR3457/2014 & Field sample & $\mathrm{SA}^{\mathrm{b}}$ & HP 2.3.4.4 & 30.29 & 29.30 & Neg & Neg & Neg & 28.34 \\
\hline 52 & A/duck/Germany/AR3465/2014 & Field sample & $\mathrm{SA}^{\mathrm{b}}$ & HP 2.3.4.4 & 23.98 & 23.15 & Neg & Neg & Neg & 20.70 \\
\hline 53 & A/duck/Germany/AR3470/2014 & Field sample & $\mathrm{SA}^{\mathrm{b}}$ & HP 2.3.4.4 & 22.15 & 23.89 & Neg & Neg & Neg & 21.78 \\
\hline
\end{tabular}

HA: haemagglutinin; HP: highly pathogenic; HPAl: highly pathogenic avian influenza; ID: identity; LP: low pathogenic; LPAI: low pathogenic avian influenza; NA: sequence not available; Neg: negative; SA: sequence available.

${ }^{a}$ Sequences were obtained from GenBank at the National Center for Biotechnology Information (NCBI) or the EpiFlu database of the Global Initiative on Sharing Avian Influenza Data (GISAID).

${ }^{b}$ Sequenced in the frame of the current study; sequences available from the authors upon request. 
2013 and 2016. Samples consisted mainly of oropharyngeal and/or cloacal swabs and tissues samples $(n=70)$ or AIV isolated from such samples $(n=36)$ (see also second table under Results).

A subsection of the 106 clinical samples $(n=13)$ was provided as dried material on Whatman FTA card (Sigma Aldrich, Germany). Samples from Western African countries were exclusively assayed at the Istituto Zooprofilattico Sperimentale delle Venezie, Padua, Italy.

\section{Design of primers and probes}

Primers were chosen based on alignments of the HA $\mathrm{H}_{5}$ gene of a selection of influenza $A$ virus sequences submitted over the past 10 years to GenBank at the National Center for Biotechnology Information (NCBI) or to the EpiFlu database of the Global Initiative on Sharing Avian Influenza Data (GISAID). Selected sequences represented Eurasian LP viruses and HP isolates and clades of the gs/GD lineage that were detected in Europe, the Middle East and Western Africa during the past decade. Selection of primers to amplify a small fragment of the HA gene spanning the endoproteolytic cleavage site aimed at being broadly inclusive so as to target as many of the published LP Eurasian $\mathrm{H}_{5} \mathrm{HA}$ sequences as possible and to distinguish them from HP viruses of the gs/GD lineage. The probes were placed directly onto the cleavage site in the attempt to specifically bind to sequences encoding either monoor polybasic patterns that distinguish LP and HP pathotypes, respectively (Table 1 ).

At first, sets of primers and probes were designed to detect and discriminate between HP and LP biotypes, i.e. Eurasian $\mathrm{H}_{5}$ viruses encoding a monobasic or a polybasic HA cleavage site. In addition, four different sets of primers and probes were developed to differentiate between gs/GD clades 2.2.1.2, 2.3.2.1 and 2.3.4.4 (A and B). Pre-selected primers were then screened in silico for their specificity properties using Shannon entropy plots implemented in the Entropy One software (http://www.hiv.lanl.gov/content/sequence/ENTROPY/entropy_one.html). Oligont (oligont) were selected so as to retain full specificity for the selected clade and to maximise entropy against all other clades. Basic physical properties of oligont were checked using the online web interface Oligo Calculator version 3.27. The finally chosen oligont are shown in Table 1. Detailed results of the in silico analyses are available on request from the authors.

\section{One-step quantitative reverse transcription PCR assays}

All reactions were performed using the AgPath-ID One-Step RT-qPCR kit (Thermofisher, scientific, United States) as follows: Reverse transcription at $45^{\circ} \mathrm{C}$ for 10 min, initial denaturation at $95^{\circ} \mathrm{C}$ for $10 \mathrm{~min}, 40$ cycles of PCR amplification at $95^{\circ} \mathrm{C}$ for $30 \mathrm{~S}, 58^{\circ} \mathrm{C}$ for $15 \mathrm{~S}$, and $72{ }^{\circ} \mathrm{C}$ for $15 \mathrm{~S}$ in a $25 \mu \mathrm{l}$ reaction mixture using $15 \mathrm{pmol}$ of each forward and reverse primers and $5 \mathrm{pmol}$ probe per reaction. For each parameter a separate reaction was used. Cycling was performed on a Biorad CFX96 Real-Time cycler (BioRad, Germany). Fluorescent signals were collected during the annealing phase, and the amplification data were analysed using Bio-Rad CFX Manager 3 software accessing automated fluorescence drift correction for baseline adjustment.

\section{Nucleotide sequencing and clade assignment} Patho- and phylotyping results obtained by newly developed RT-qPCRs were counter-checked by nt (nt) sequencing of the entire or parts of the $\mathrm{HA}$ gene of the respective isolates/clinical samples. Amplification of the HA gene was performed using primers published previously [24] and primers recommended in the European Union Diagnostic Manual for Al in a one-step RT-PCR [25]. In addition, amplificates of the HPAI $\mathrm{H}_{5}$ and LPAI H5 RT-qPCRs were used for sequencing purposes as well. Products were size-separated in agarose gels, excised and purified using the QIAquick Gel Extraction Kit (Qiagen, Hilden, Germany). Purified $P C R$ products were used for cycle sequencing reactions (BigDye Terminator v1.1 Cycle Sequencing Kit, Applied Biosystems, California, United States) the products of which were purified using NucleoSEQ columns (Macherey-Nagel GmbH and Co, Düren, Germany) and sequenced on an ABI PRISM 3130 Genetic Analyzer (Life Technologies, Darmstadt, Germany).

For pathotyping, deduced amino acid sequences of the endoproteolytical cleavage site of the HA gene were inspected and compared with the molecular pathotyping database provided by OFFLU [26]. Assignment of nt sequences to the gs/GD HPAI $\mathrm{H}_{5}$ virus clade system was performed by use of clade prediction tool implemented in the Influenza Research Database [27].

\section{Results}

Analytical specificity of pathotyping and phylotyping quantitative reverse transcription PCR assays

The specificity of the assays was evaluated with viral RNA from representative influenza A subtype $\mathrm{H}_{5}$ viruses that had been phylotyped based on full-length $\mathrm{HA}$ nt sequence analysis (Table 2). Furthermore, non- $\mathrm{H}_{5}$ subtypes, i.e. $\mathrm{H}_{9} \mathrm{~N}_{2}$ and $\mathrm{H}_{7} \mathrm{~N}_{7}$, as well as non-influenza avian viruses i.e. avian infectious bronchitis virus (IBV) and Newcastle disease virus (NDV) were employed (Table 2), and none of them was detected by any of the specific PCRs.

In the initial evaluation of the specificity of the pathotyping RT-qPCR assays carried out using two reference viruses: HPAI A/chicken/Egypt/AR236/2015 $\left(\mathrm{H}_{5} \mathrm{~N}_{1}\right.$, clade 2.2.1.2) and LPAI A/turkey/Germany/R2025/2008 $\left(\mathrm{H}_{5} \mathrm{~N}_{3}\right)$, specific reactivity exclusively with the homopathotypic virus was evident. In a second step, assays were extended to the full range of 24 reference viruses yielding a similar sharp distinction between HP and LP cleavage sites (Table 2). 


\section{TABLE 3B}

Pathotyping and phylotyping of different potentially zoonotic HPAI and LPAI influenza A subtype H5 virus isolates and field samples collected from poultry and wild bird species in different countries, 2013-2016

\begin{tabular}{|c|c|c|c|c|c|c|c|c|c|c|}
\hline \multirow{2}{*}{ No. } & \multirow{2}{*}{ Sample ID } & \multirow{2}{*}{ Type of sample } & \multirow{2}{*}{$\begin{array}{l}\text { Accession } \\
\text { Number }^{\mathrm{a}}\end{array}$} & \multirow{2}{*}{ Clade } & \multicolumn{6}{|c|}{ PCR results } \\
\hline & & & & & M1.2 & $\mathrm{HPAI}_{5}$ & LPAI H5 & 2.2 .1 .2 & 2.3.2.1 & 2.3.4.4 \\
\hline 54 & A/wild-duck/Germany/AR8603/2016 & Field sample & $\mathrm{SA}^{\mathrm{b}}$ & HP 2.3.4.4b & 22.51 & 22.90 & Neg & Neg & Neg & 21.14 \\
\hline 55 & A/greyleg goose/Germany/AR8604/2016 & Field sample & $S A^{b}$ & HP 2.3.4.4b & 22.41 & 22.30 & Neg & Neg & $\mathrm{Neg}$ & 20.26 \\
\hline 56 & A/greater scaup/Germany/AR909o/2016 & Field sample & $S A^{b}$ & HP 2.3.4.4b & 27.29 & 31.71 & $\mathrm{Neg}$ & Neg & Neg & 29.61 \\
\hline 57 & A/greater scaup/Germany/AR9091/2016 & Field sample & $\mathrm{SA}^{\mathrm{b}}$ & HP 2.3.4.4b & 28.95 & 34.40 & $\mathrm{Neg}$ & Neg & $\mathrm{Neg}$ & 31.74 \\
\hline 58 & A/greater scaup/Germany/AR9092/2016 & Field sample & $\mathrm{SA}^{\mathrm{b}}$ & HP 2.3.4.4b & 23.85 & 25.70 & Neg & Neg & $\mathrm{Neg}$ & 26.05 \\
\hline 59 & A/grey heron/Germany/AR9093/2016 & Field sample & $\mathrm{SA}^{\mathrm{b}}$ & HP 2.3.4.4b & 20.10 & 22.62 & Neg & Neg & $\mathrm{Neg}$ & 22.44 \\
\hline 60 & A/greater scaup/Germany/AR9094/2016 & Field sample & $\mathrm{SA}^{\mathrm{b}}$ & HP 2.3.4.4b & 16.31 & 20.62 & $\mathrm{Neg}$ & Neg & Neg & 18.92 \\
\hline 61 & A/greater scaup/Germany/AR9095/2016 & Field sample & $\mathrm{SA}^{\mathrm{b}}$ & HP 2.3.4.4b & 20.99 & 22.79 & $\mathrm{Neg}$ & Neg & $\mathrm{Neg}$ & 21.75 \\
\hline 62 & A/northern pintail/Germany/AR9096/2016 & Field sample & $\mathrm{SA}^{\mathrm{b}}$ & HP 2.3.4.4b & 22.83 & 28.12 & $\mathrm{Neg}$ & Neg & $\mathrm{Neg}$ & 23.95 \\
\hline 63 & A/bean goose/Germany/AR9097/2016 & Field sample & $\mathrm{SA}^{\mathrm{b}}$ & HP 2.3.4.4b & 22.97 & 24.88 & Neg & Neg & Neg & 24.92 \\
\hline 64 & A/herring gull/Germany/AR9098/2016 & Field sample & $\mathrm{SA}^{\mathrm{b}}$ & HP 2.3.4.4b & 20.40 & 22.35 & Neg & Neg & Neg & 23.64 \\
\hline 65 & A/mute swan/Germany/AR9099/2016 & Field sample & $\mathrm{SA}^{\mathrm{b}}$ & HP 2.3.4.4b & 21.12 & 25.83 & $\mathrm{Neg}$ & $\mathrm{Neg}$ & Neg & 22.75 \\
\hline 66 & A/chicken/Germany/AR9140/2016 & Field sample & $\mathrm{SA}^{\mathrm{b}}$ & HP 2.3.4.4b & 21.08 & 20.55 & $\mathrm{Neg}$ & Neg & $\mathrm{Neg}$ & 22.92 \\
\hline 67 & A/chicken/Germany/AR9141/2016 & Field sample & $\mathrm{SA}^{\mathrm{b}}$ & HP 2.3.4.4b & 21.21 & 20.13 & $\mathrm{Neg}$ & $\mathrm{Neg}$ & $\mathrm{Neg}$ & 23.12 \\
\hline 68 & A/chicken/Germany/AR9143/2016 & Field sample & $S A^{b}$ & HP 2.3.4.4b & 32.27 & 31.51 & $\mathrm{Neg}$ & Neg & $\mathrm{Neg}$ & 35.49 \\
\hline 69 & A/chicken/Germany/AR9144/2016 & Field sample & $\mathrm{SA}^{\mathrm{b}}$ & HP 2.3.4.4b & 21.89 & 20.89 & $\mathrm{Neg}$ & Neg & Neg & 25.92 \\
\hline 70 & A/chicken/Italy/22/1998 & Isolate & CAP $58165_{5}$ & $\mathrm{LPAI}_{5} \mathrm{~N}_{9}$ & 12.34 & $\mathrm{Neg}$ & 19.06 & Neg & $\mathrm{Neg}$ & $\mathrm{Neg}$ \\
\hline 71 & $\mathrm{~A} / \mathrm{mallard} /$ Germany/Wv1349-51K/2003 & Isolate & $\mathrm{CAP}_{5} 8164$ & LPAI $\mathrm{H}_{5} \mathrm{~N}_{3}$ & 26.00 & Neg & 14.79 & Neg & Neg & Neg \\
\hline 72 & A/mallard/Germany/Wv476/2004 & Isolate & NA & LPAI $\mathrm{H}_{5} \mathrm{~N}_{2}$ & 29.87 & Neg & 29.5 & Neg & Neg & Neg \\
\hline 73 & A/mallard/Germany/Wv474-77K/2004 & Isolate & NA & LPAI $\mathrm{H}_{5} \mathrm{~N}_{2}$ & 29.64 & Neg & 34.81 & Neg & Neg & Neg \\
\hline 74 & A/ostrich/Germany/R5-10/2006 & Isolate & $\mathrm{HF}_{563057}$ & LPAI $\mathrm{H}_{5} \mathrm{~N}_{3}$ & 26.80 & $\mathrm{Neg}$ & 26.19 & Neg & $\mathrm{Neg}$ & Neg \\
\hline 75 & A/mallard/Germany/R2557/2006 & Isolate & NA & LPAI $\mathrm{H}_{5} \mathrm{~N}_{3}$ & 26.24 & Neg & 27.61 & Neg & $\mathrm{Neg}$ & Neg \\
\hline 76 & $\mathrm{~A} /$ mallard/Germany/R731/2008 & Isolate & $S A^{b}$ & LPAI $\mathrm{H}_{5} \mathrm{~N}_{3}$ & 30.36 & Neg & 32.30 & Neg & $\mathrm{Neg}$ & Neg \\
\hline 77 & $\mathrm{~A} /$ mallard/Germany/R771/2008 & Isolate & $S A^{b}$ & LPAI $\mathrm{H}_{5} \mathrm{~N}_{3}$ & 29.24 & Neg & 30.28 & Neg & Neg & Neg \\
\hline 78 & $\mathrm{~A} /$ mallard/Germany/R772/2008 & Isolate & $S A^{b}$ & LPAI $\mathrm{H}_{5} \mathrm{~N}_{3}$ & 23.68 & Neg & 24.50 & Neg & Neg & $\mathrm{Neg}$ \\
\hline 79 & A/turkey/Germany/R1550/2008 & Isolate & NA & $\mathrm{LPAI}_{5} \mathrm{~N}_{3}$ & 25.17 & Neg & 26.22 & Neg & Neg & Neg \\
\hline 80 & A/turkey/Germany/R1551/2008 & Isolate & NA & LPAI $\mathrm{H}_{5} \mathrm{~N}_{3}$ & 24.03 & Neg & 24.91 & Neg & Neg & $\mathrm{Neg}$ \\
\hline 81 & A/turkey/Germany/R1557/2008 & Isolate & $\mathrm{SA}^{\mathrm{ba}}$ & $\mathrm{LPAI}_{5} \mathrm{~N}_{3}$ & 23.50 & $\mathrm{Neg}$ & 24.16 & $\mathrm{Neg}$ & $\mathrm{Neg}$ & Neg \\
\hline 82 & A/turkey/Germany/R1612/2008 & Isolate & NA & LPAI $\mathrm{H}_{5} \mathrm{~N}_{3}$ & 27.43 & Neg & 29.25 & $\mathrm{Neg}$ & $\mathrm{Neg}$ & Neg \\
\hline 83 & A/turkey/Germany/R2014/2008 & Isolate & $\mathrm{SA}^{\mathrm{b}}$ & LPAI $\mathrm{H}_{5} \mathrm{~N}_{3}$ & 24.33 & $\mathrm{Neg}$ & 21.99 & $\mathrm{Neg}$ & $\mathrm{Neg}$ & Neg \\
\hline 84 & A/turkey/Germany/R2015/2008 & Isolate & $S A^{b}$ & LPAI $\mathrm{H}_{5} \mathrm{~N}_{3}$ & 15.69 & $\mathrm{Neg}$ & 30.13 & $\mathrm{Neg}$ & $\mathrm{Neg}$ & Neg \\
\hline 85 & A/turkey/Germany/R2016/2008 & Isolate & $S A^{b}$ & LPAI $\mathrm{H}_{5} \mathrm{~N}_{3}$ & 18.13 & $\mathrm{Neg}$ & 15.96 & $\mathrm{Neg}$ & $\mathrm{Neg}$ & Neg \\
\hline 86 & A/turkey/Germany/R2017/2008 & Isolate & $S A^{b}$ & LPAI $\mathrm{H}_{5} \mathrm{~N}_{3}$ & 18.32 & Neg & 16.92 & Neg & $\mathrm{Neg}$ & $\mathrm{Neg}$ \\
\hline 87 & A/turkey/Germany/R2018/2008 & Isolate & $S A^{b}$ & $\mathrm{LPAI}_{5} \mathrm{~N}_{3}$ & 14.16 & Neg & 16.05 & Neg & Neg & Neg \\
\hline 88 & A/turkey/Germany/R2019/2008 & Isolate & $S A^{b}$ & $\mathrm{LPAI}_{5} \mathrm{~N}_{3}$ & 14.55 & Neg & 16.84 & Neg & Neg & Neg \\
\hline 89 & A/turkey/Germany/R2020/2008 & Isolate & $S A^{b}$ & LPAI $\mathrm{H}_{5} \mathrm{~N}_{3}$ & 19.38 & Neg & 16.73 & Neg & Neg & Neg \\
\hline 90 & A/turkey/Germany/R2021/2008 & Isolate & $S A^{b}$ & LPAI $\mathrm{H}_{5} \mathrm{~N}_{3}$ & 12.71 & $\mathrm{Neg}$ & 13.51 & $\mathrm{Neg}$ & $\mathrm{Neg}$ & Neg \\
\hline 91 & A/turkey/Germany/R2022/2008 & Isolate & $\mathrm{SA}^{\mathrm{b}}$ & LPAI $\mathrm{H}_{5} \mathrm{~N}_{3}$ & 12.63 & Neg & 13.18 & $\mathrm{Neg}$ & $\mathrm{Neg}$ & Neg \\
\hline 92 & A/turkey/Germany/R2023/2008 & Isolate & $\mathrm{SA}^{\mathrm{b}}$ & LPAI $\mathrm{H}_{5} \mathrm{~N}_{3}$ & 19.37 & $\mathrm{Neg}$ & 17.07 & $\mathrm{Neg}$ & $\mathrm{Neg}$ & Neg \\
\hline 93 & A/turkey/Germany/R2024/2008 & Isolate & $S A^{b}$ & LPAI $\mathrm{H}_{5} \mathrm{~N}_{3}$ & 22.52 & Neg & 20.39 & Neg & $\mathrm{Neg}$ & $\mathrm{Neg}$ \\
\hline 94 & A/turkey/Germany/R2025/2008 & Isolate & $S A^{b}$ & LPAI $\mathrm{H}_{5} \mathrm{~N}_{3}$ & 22.44 & Neg & 25.22 & Neg & $\mathrm{Neg}$ & Neg \\
\hline 95 & A/turkey/Germany/R2026/2008 & Isolate & $S A^{b}$ & $\mathrm{LPAI}_{5} \mathrm{~N}_{3}$ & 14.70 & Neg & 16.26 & Neg & $\mathrm{Neg}$ & Neg \\
\hline 96 & A/turkey/Germany/R2027/2008 & Isolate & $\mathrm{SA}^{\mathrm{b}}$ & LPAI $\mathrm{H}_{5} \mathrm{~N}_{3}$ & 17.80 & Neg & 16.06 & $\mathrm{Neg}$ & Neg & Neg \\
\hline 97 & A/mallard/Germany/R2892-94/2009 & Isolate & $\mathrm{EPI}_{356412}$ & $\mathrm{LPAI}_{5} \mathrm{~N}_{3}$ & 11.98 & Neg & 14.37 & $\mathrm{Neg}$ & Neg & Neg \\
\hline 98 & A/duck/Germany/AR1965/2013 & Field sample & NA & LPAI $\mathrm{H}_{5} \mathrm{~N}_{3}$ & 26.62 & Neg & 27.25 & $\mathrm{Neg}$ & $\mathrm{Neg}$ & $\mathrm{Neg}$ \\
\hline 99 & A/turkey/Germany/AR1892/1/2014 & Field sample & $\mathrm{SA}^{\mathrm{b}}$ & LPAI $\mathrm{H}_{5} \mathrm{~N}_{2}$ & 20.03 & $\mathrm{Neg}$ & 21.15 & Neg & $\mathrm{Neg}$ & Neg \\
\hline 100 & A/duck/Germany/AR1/2015 & Field sample & $\mathrm{SA}^{\mathrm{b}}$ & LPAI $\mathrm{H}_{5} \mathrm{~N}_{3}$ & 29.20 & $\mathrm{Neg}$ & 34.01 & $\mathrm{Neg}$ & $\mathrm{Neg}$ & Neg \\
\hline 101 & A/swan/Germany/AR111/2015 & Field sample & $\mathrm{SA}^{\mathrm{b}}$ & LPAI $\mathrm{H}_{5} \mathrm{~N}_{4}$ & 27.45 & Neg & 31.02 & Neg & $\mathrm{Neg}$ & Neg \\
\hline 102 & A/goose/Germany/AR398/2015 & Field sample & $S A^{b}$ & LPAI & 31.09 & Neg & 33.69 & Neg & Neg & $\mathrm{Neg}$ \\
\hline 103 & A/duck/Germany/AR1231/1/2015 & Field sample & NA & LPAI $\mathrm{H}_{5} \mathrm{~N}_{2}$ & 26.74 & Neg & 32.91 & Neg & $\mathrm{Neg}$ & Neg \\
\hline 104 & A/duck/Germany/AR2853/15-1/2015 & Field sample & $S A^{b}$ & $\mathrm{LPAI}_{5} \mathrm{~N}_{3}$ & 27.06 & Neg & 26.25 & $\mathrm{Neg}$ & $\mathrm{Neg}$ & Neg \\
\hline 105 & A/goose/Germany/AR3264/1/2015 & Field sample & $\mathrm{SA}^{\mathrm{b}}$ & LPAI $\mathrm{H}_{5} \mathrm{~N}_{2}$ & 34.47 & Neg & 35.50 & $\mathrm{Neg}$ & Neg & Neg \\
\hline 106 & A/wild bird/Germany/AR221/2015 & Field sample & $\mathrm{SA}^{\mathrm{b}}$ & $\mathrm{LPH}_{5} \mathrm{~N}_{3}$ & 22.17 & Neg & 23.48 & Neg & $\mathrm{Neg}$ & $\mathrm{Neg}$ \\
\hline
\end{tabular}

HA: haemagglutinin; HP: highly pathogenic; HPAl: highly pathogenic avian influenza; ID: identity; LP: low pathogenic; LPAI: low pathogenic avian influenza; NA: sequence not available; Neg: negative; SA: sequence available.

a Sequences were obtained from GenBank at the National Center for Biotechnology Information (NCBI) or the EpiFlu database of the Global Initiative on Sharing Avian Influenza Data (GISAID).

b Sequenced in the frame of the current study; sequences available from the authors upon request. 
Primers and probes for phylotyping RT-qPCR assays distinguishing three clades of gs/GD origin HPAIV $\mathrm{H}_{5}$ were placed within the HA1-fragment of the HA gene. This region encodes the receptor binding unit and harbours a number of neutralisation-relevant epitopes that are targets of antigenic drift. Hence, the HA1 fragment harbours regions that are the least conserved within the influenza A virus genome. Primer selection aimed at the inclusion of as many as possible distinguishing nt that would define exclusivity at the five most 3' positions while probes were placed so as to accommodate distinguishing $n t$ in the centre of the oligont. In order not to compromise amplification efficacy, amplicon size was limited to $130 \mathrm{nt}$ wherever possible given the above mentioned constraints for primers and probes. The finally chosen oligont are listed in Table 1 and provided specific detection exclusively of the homologous clade. No cross-reactivity among the other gs/GD clades examined was evident on basis of the used panel of reference viruses (Table 2). Also, no cross-reactivity was detected for any of the five assays against other influenza $A$ viruses or other avian viral respiratory pathogens (Table 2 ).

\section{Validation of the analytical sensitivity, limit of} detection and precision

Detection limits of the assays were determined by testing 10 -fold serial dilutions of viral RNA extracted from representative viruses of each of the three HPAI virus clades (2.2.1.2, 2.3.2.1 and 2.3.4.4), and of Eurasian $\mathrm{H}_{5} \mathrm{LPAl}$ virus. Cycle of quantification $(\mathrm{Cq})$ values were compared with a standard RT-qPCR for the matrix (M) gene of these viruses with a reported detection limit of 2 to 20 RNA copies/5 $\mu \mathrm{l}$ [28]. Average values of three separate runs were computed and plotted using SigmaPlot $V 11$ software. Plotting these values revealed a linear relationship between the log of the viral RNA dilution and the $\mathrm{Cq}$ value for all assays and the kinetics of the assays and their sensitivity were determined to be very similar to the generic M gene RT-qPCR (M1.2 RT-qPCR [29]) (Figure 1).

The correlation coefficient of the standard curves was 0.99 for all assays, indicating a highly precise log-linear relationship between the viral RNA log dilution and the corresponding Cq-value (Figure 1). Based on these results the threshold distinguishing positive and negative was set at $\mathrm{Cq}=38$.

\section{Pathotyping and phylotyping of clinical} samples of potentially zoonotic Eurasian avian influenza A subtype $\mathrm{H} 5$ viruses by quantitative reverse transcription PCR

In order to evaluate the diagnostic performance capacity of the developed assays, field samples (RNA extracted from swabs, tissues or FTA cards) and clinical virus isolates obtained during the period 2013 to 2016 (HPAl viruses) or 2003 to 2015 (LPAl viruses) were examined. The sample set was preselected on basis of a positive generic M-specific RT-qPCR.
Among the final set of 106 samples, the pathotyping RT-qPCRs sharply discerned two groups of 69 samples reacting only in the new HPAI H5 RT-qPCR while 37 samples reacted positive in the LPAI $\mathrm{H}_{5} \mathrm{RT}-\mathrm{qPCR}$ (Figure 2a; Table 3).

All pathotyping results matched the results obtained by nt sequence analysis of the HA cleavage site. However, in a few samples (two isolates, 10 clinical samples) of HP viruses, the LPAI $\mathrm{H}_{5}$ RT-qPCR also gave a weak positive signal $(\mathrm{Cq}>35)$. Compared with the LPAI $\mathrm{H}_{5}$ signal the HPAI $\mathrm{H}_{5}$ signal of these samples yielded $\mathrm{Cq}$ values 6-10 units lower on average ascertaining good diagnostic specificity. Depending on the clade, the HP phenotype was detected with equal (clade 2.3.2.1) or slightly reduced (clade 2.2 .1 .2 ) sensitivity; the $\mathrm{LP} \mathrm{H}_{5}$ RT-qPCR appeared to be slightly less sensitive than the M PCR as far as clinical samples were concerned (Table 3; Figure $2 \mathrm{a}$ and $\mathrm{c}$ ). Sequences across the cleavage sites of these samples are presented in a supplemental alignment (Figure 2).

In a next step, the samples that were designated HPAI $\mathrm{H}_{5}$-positive were subjected to the three phylotyping RT-qPCRs. Here, 15, 21 and 33 samples, respectively, were exclusively positive for either clade 2.2.1.2, 2.3.2.1 or 2.3.4.4 (Table 3). Thus, a clear cut clade assignment was possible for all gs/GD HP $\mathrm{H}_{5}$ samples. Results were counterchecked by feeding available HA sequences of these samples into the IRD clade prediction tool (www.fludb.org/brc/h5n1-Classifier.spg?met hod=ShowCleanInputPage\&decorator=influenz): In all cases the same clade was assigned by sequence analysis and by PCR. In a final step also all LPAI $\mathrm{H}_{5}$ samples were tested in the phylotyping RT-qPCRs and none of them cross-reacted. Regarding the sensitivity of these PCRs, the $\mathrm{Cq}$ values were compared with those of the generic M1.2-specific RT-qPCR (Figure 2b). For clade 2.2.1.2 and 2.3.2.1 the sensitivity was almost identical to the M PCR; for clade 2.3.4.4a, the clade-specific PCR proved to be slightly more sensitive while viruses of clade 2.3.4.4b were detected at a slightly lower sensitivity; detection of clade 2.3.4.4b viruses was slightly less sensitive than the M PCR (Figure $2 \mathrm{~b}$ and c; Table 3 ) as far as clinical samples were concerned.

Rank Sum tests implemented in the SigmaPlot software package were performed and no statistically significant difference between the median $\mathrm{Cq}$ values of each specific assay and the M1.2 RT-qPCR assay was found $(p>0,50)$ indicating that the newly developed RT-qPCRs display similar analytical sensitivity. Thus, the phylotyping RT-qPCRs allow a sensitive and highly specific detection and distinction of the three major gs/GD clades currently circulating in countries where the viruses were obtained from.

\section{Discussion}

Rapid molecular diagnosis including patho- and phylotyping is basis to enable measures aimed at repressing 
the spread of potentially zoonotic HPAl viruses. The TaqMan PCR technology has proven reliable, versatile, and comparatively cost-effective in the generic detection and subtype differentiation of AIV [30]. Further differentiation of clades, lineages and pathotypes was previously nearly entirely based on nt sequencing approaches which require expensive equipment and are time consuming. In epidemiologically complex settings where different lineages and pathotypes of potentially zoonotic and notifiable infectious agents co-circulate, a more rapid and direct access to testing and results, e.g. by using RT-qPCRs, is desirable. Although RT-qPCRs are inferior to sequencing techniques in terms of retrievable data details, they are superior with respect to time-to-diagnosis and ease-ofuse. This concept which we used earlier for pathotyping of $\mathrm{H}_{5} \mathrm{~N}_{1}$ [31], was here further extended and refined for the identification and discrimination of avian influenza A subtype $\mathrm{H}_{5}$ viruses of different patho- and phylotypes. The focus was put on those clades of $\mathrm{H}_{5}$ viruses $(2.2 .1 .2,2.3 .2 .1,2.3 .4 .4)$ that had previously 'escaped' from Asia and were detected in western parts of Eurasia and in Africa.

Pathotyping of avian influenza A subtype $\mathrm{H}_{5}$ viruses is mandatory from an animal health perspective. The pathotyping RT-qPCRs presented here reduce time-to-diagnosis to just three hours following sample receipt. To our knowledge this is the broadest and most detailed attempt of AIV pathotyping using RT-qPCR. The availability of highly sensitive pathotyping PCRs would also allow to detect mixtures of HP and $\mathrm{LP} \mathrm{H}_{5}$ viruses in the same sample; in fact, some of our HP-positive field samples also gave weak LP signals (Table 3, sample numbers 2, 9, 10, 14, 15, 30, 32, $35,37,42-5)$. Yet, LPAI pathotypes in these samples were detected at distinctly higher $\mathrm{Cq}$ values indicating either a minor population in a quasispecies of different pathotypes or expressing some cross-reactivity of LPAI primers and probe; in any case, the detection of HPAI genotypes as a major population in a set of field samples was always unequivocal. Further insight into the true nature of these mixtures would only be unravelled by deep sequencing approaches of those samples.

Rapid pathotyping enables rapid implementation of appropriate measures to prevent further spread of virus such as closure of poultry holdings and/or live poultry markets, culling of infected flocks etc. This impedes accumulation of potentially zoonotic AIV at the poultry-human interface which in turn lowers the risks of human infection.

Phylotyping of gs/GD HPAI $\mathrm{H}_{5}$ virus clades is important since each clade, and often also sublineages thereof, display distinct antigenic and pathogenetic properties. This has direct implications, as by assigning the matching clade, appropriate vaccines that ensure the closest antigenic match with the circulating viruses can be selected [32,33]. In particular, countries where gs/GD viruses have become endemic in poultry populations, rely on vaccination of poultry on a broad scale to suppress circulating viruses and to limit risks of human exposure [23]. However, it should be noted that mutant escape variants within these clades selected by vaccine-induced population immunity will not be detected as such by the assays, and in fact, such mutants may also be detected at lower sensitivity if primer and/ or probe binding sites are affected by mutations. Detection of variants will still depend on either nt sequencing or virus isolation/antigenic characterisation approaches but the newly developed assays will aid in selection of meaningful samples in this respect. In particular, samples that do not give conclusively similar Cq values in the generic and the specific assays should prompt in-depth analysis by nt sequencing.

It should be clearly stated that the assays presented here have limitations owed to the restricted geographical distribution of the targeted clades. The use of the newly developed PCRs in regions where viruses belonging to the targeted clades $(2.2 .1 .2,2.3 .2 .1 \mathrm{C}$ and 2.3.4.4) are reportedly absent is only recommended if immediate incursions with any of these clades are apprehended. Phylotyping indirectly may point towards zoonotic potential since different gs/GD lineages vary in their zoonotic propensity: Egyptian 2.2.1.2 viruses are characterised by increased affinity to human-like sialic acid receptors and have caused by far the largest number of human influenza $\mathrm{A}\left(\mathrm{H}_{5} \mathrm{~N}_{1}\right)$ virus infections over the past decade [12]. For clade 2.3.2.1C viruses, repeatedly detected in the Middle East (excluding Egypt) and endemic in Western African countries, only few human cases have been recorded. The 2.3.4.4 viruses currently present in various parts of Europe have not provoked human infection so far [34].

Extended co-circulation of more than one gs/GD lineage in poultry and/or wild birds in a wider geographic region was repeatedly reported $[35,36]$. It is pivotal, for the above mentioned reasons, to detect incursions of distinct HPAl virus lineages in a timely manner. In this respect, the newly developed RT-qPCR assays were shown to be useful tools for an improved rapid and simple characterisation of patho- and phylotypes of Eurasian origin avian influenza $\mathrm{A}$ subtype $\mathrm{H}_{5}$ viruses. The assays aid in speeding up diagnosis on clinical samples because the time consuming (initial) need of virus isolation and nt sequencing is avoided. Given the high substitution rate of $\mathrm{HP} \mathrm{H}_{5}$ influenza viruses frequent checks and, if required, updates of the primers and probes are recommended to ensure full specificity and sensitivity of the patho- and phylotyping RT-qPCRs. These PCRs are advantageous in particular for wild bird samples, especially those that contain LPAI viruses, often with low viral loads and therefore fail to yield replication-competent virus. With respect to HPAI virus, the renouncement from initial virus isolation improves biosecurity. However, the presented assays are not intended to replace virus isolation and antigenic characterisation as a means to detect emerging antigenic drift mutants. Nevertheless, they may aid in selection 
of appropriate samples for such tasks. Accurate phylotyping also facilitates selection of appropriate vaccines as it serves as an early warning for the incursion of new and antigenically possibly distinct phylotypes.

\section{Conclusions}

The assays reported here are primarily intended for screening purposes of avian samples; confirmatory assays, including nt sequence analyses and antigenic characterisation, are still required for new incursions and outbreak scenarios that feature an expansion of the geographic area and/or the range of affected species or poultry sectors. When used in the frame of on-going outbreaks, in particular in regions where vaccination is not used as a preventive measure, results of the patho- and phylotyping PCRs are deemed solid enough for reporting purposes and to justify the implementation of restriction measures. In such settings, similar to the current outbreaks of clade 2.3.4.4b $\mathrm{HP} \mathrm{H}{ }_{5} \mathrm{~N} 8$ in Europe, the assays can be prioritised to running the HP and only one (i.e. the fitting) of the phylotyping PCRs on M1.2- and $\mathrm{H}_{5}$ PCR-positive samples. This significantly speeds up time-to-diagnosis and reduces reaction times in a OneHealth approach of repressing the spread of gs/GD HP AIV. Sequencing facilities, classically required for patho- and phylotyping, may not be available, and even not logistically accessible in many regions severely affected by $\mathrm{H}_{5} \mathrm{HPAl}$ incursions. The prospect of having sequencing-independent, TaqMan-based specific and sensitive typing assays, as described here, available in developing regions is expected to boost regional diagnostic capacities eventually leading to improved disease control.

\section{Acknowledgements}

The authors greatly acknowledge Dr Joseph Adongo Awuni from Accra Veterinary Laboratory, Veterinary Services Directorate, Ministry of Food and Agriculture, Accra (Ghana), Dr Germaine L. Minoungou from the Laboratoire National d'Elevage in Ouagadougou (Burkina Faso), Dr Zangui Ibrahima Mahaman Sani and Dr Abdou Alassane from the LABOCEL - Laboratoire Central de l'Elevage, Niamey (Niger), Dr Emmanuel Couacy-Hymann from the Laboratoire Central de Pathologie Animale, Bingerville (Côte d'Ivoire) and Abel Wade from the National Veterinary Laboratory (LANAVET), Yaounde (Cameroon) for submitting samples to the Istituto Zooprofilattico Sperimentale delle Venezie, Padova, Italy and for their interest in collaborating in the validation of the developed real-time RT-PCR assays. Field samples from Ghana, Côte d'Ivoire, Cameroon, Niger, Burkina Faso used to evaluate the diagnostic performance capacity of the developed assays were obtained through technical support provided by the United Nations Food and Agriculture Organisation (letter of agreement number 321149).

We wish to thank Aline Maksimov and Diana Wessler for excellent technical support. We are grateful to veterinarians and diagnosticians in Germany for submitting diagnostic samples.

We also gratefully acknowledge the authors, originating and submitting laboratories of the sequences from GISAID's EpiFlu Database used in this study; a detailed list of the respective sequences from the EpiFlu database is available on request from the authors.
Conflict of interest

None declared. One of the authors (N.A.) works for Boehringer Ingelheim, Dubai, United Arab Emirates.

\section{Authors' contributions}

Mahmoud M. Naguib, Annika Graaf and Timm Harder conceived the study. Andrea Fortin, Ulrich Wernery, Nadim Amarin, Hussein A. Hussein, Hesham Sultan and Basem Al Adhadh were involved in the collection, initial analysis and provision of viruses and field samples. Christine Luttermann conducted the Sanger sequencing analyses. Mahmoud $M$. Naguib, Annika Graaf and Andrea Fortin produced, analysed and interpreted data. Timm Harder and Mahmoud M. Naguib drafted the manuscript. Isabella Monne, Martin Beer and all co-authors critically analysed and revised the manuscript and provided final approval.

\section{References}

1. Cheung TK, Poon LL. Biology of influenza a virus.Ann N Y Acad Sci. 2007;1102(1):1-25. DOI: 10.1196/annals.1408.001 PMID: 17470908

2. Webster RG, Govorkova EA. Continuing challenges in influenza. Ann N Y Acad Sci. 2014;1323(1):115-39. DOI: 10.1111/nyas.12462 PMID: 24891213

3. França MS, Brown JD. Influenza pathobiology and pathogenesis in avian species.Curr Top Microbiol Immunol. 2014;385:221-42. DOI: 10.1007/82_2014_385 PMID: 25015786

4. Wallace RG, Bergmann L, Kock R, Gilbert M, Hogerwerf L, Wallace R, et al. The dawn of Structural One Health: a new science tracking disease emergence along circuits of capital. Soc Sci Med. 2015;129:68-77. DOI: 10.1016/j. socscimed.2014.09.047 PMID: 25311784

5. Malik Peiris JS. Avian influenza viruses in humans. Rev Sci Tech. 2009;28(1):161-73. DOI: 10.20506/rst.28.1.1871 PMID: 19618624

6. Capua I, Munoz O. Emergence of influenza viruses with zoonotic potential: open issues which need to be addressed. A review.Vet Microbiol. 2013;165(1-2):7-12. DOI: 10.1016/j. vetmic.2013.01.044 PMID: 23567150

7. Smith GJ, Donis RO. World Health Organization/World Organisation for Animal HF, Agriculture Organization HEWG. Nomenclature updates resulting from the evolution of avian influenza $\mathrm{A}\left(\mathrm{H}_{5}\right)$ virus clades 2.1.3.2a, 2.2.1, and 2.3.4 during 2013-2014. Influenza Other Respi Viruses. 2015;9(5):271-6. DOI: 10.1111/irv.12324

8. Lycett S, Bodewes R, Pohlmann A, Banks J, Bányai K, Boni $\mathrm{MF}$, et al. , Global Consortium for $\mathrm{H}_{5} \mathrm{~N} 8$ and Related Influenza Viruses. Role for migratory wild birds in the global spread of avian influenza H5N8. Science. 2016;354(6309):213-7. DOI: 10.1126/science.aaf8852 PMID: 27738169

9. Adlhoch C, Gossner C, Koch G, Brown I, Bouwstra R, Verdonck $F$, et al. Comparing introduction to Europe of highly pathogenic avian influenza viruses $\mathrm{A}\left(\mathrm{H}_{5} \mathrm{~N} 8\right)$ in 2014 and $A\left(\mathrm{H}_{5} \mathrm{~N}_{1}\right)$ in 2005. Euro Surveill. 2014:19(50):20996. DOI. 10.2807/1560-7917.ES2014.19.50.20996 PMID: 25597538

10. Abdelwhab EM, Hassan MK, Abdel-Moneim AS, Naguib MM, Mostafa A, Hussein IT, et al. Introduction and enzootic of A/ $\mathrm{H}_{5} \mathrm{~N}_{1}$ in Egypt: Virus evolution, pathogenicity and vaccine efficacy ten years on. Infect Genet Evol. 2016;40:80-90. DOI: 10.1016/j.meegid.2016.02.023 PMID: 26917362

11. World Health Organization (WHO). Cumulative number of confirmed human cases for avian influenza $\mathrm{A}\left(\mathrm{H}_{5} \mathrm{~N}_{1}\right)$ reported to WHO, 2003-2016. Geneva: WHO; 2016. [Accessed 01 Jan 2017]. Available from: http://www.who.int/influenza/ human_animal_interface/ $\mathrm{H}_{5} \mathrm{~N} 1$ _cumulative_table_archives/ en/.

12. Arafa AS, Naguib MM, Luttermann C, Selim AA, Kilany WH, Hagag N, et al. Emergence of a novel cluster of influenza $A\left(\mathrm{H}_{5} \mathrm{~N}_{1}\right)$ virus clade 2.2 .1 .2 with putative human health impact in Egypt, 2014/15. Euro Surveill. 2015;20(13):2-8. DOI: 10.2807/1560-7917.ES2015.20.13.21085 PMID: 25860390

13. Tosh C, Nagarajan S, Kumar M, Murugkar HV, Venkatesh G, Shukla S, et al. Multiple introductions of a reassortant $\mathrm{H}_{5} \mathrm{~N}_{1}$ avian influenza virus of clade 2.3.2.1C with $\mathrm{PB} 2$ gene of $\mathrm{H}_{9} \mathrm{~N}_{2}$ subtype into Indian poultry. Infect Genet Evol. 2016;43:173-8. DOI: 10.1016/j.meegid.2016.05.012 PMID: 27174088 
14. Bi Y, Chen J, Zhang Z, Li M, Cai T, Sharshov K, et al. Highly pathogenic avian influenza $\mathrm{H}_{5} \mathrm{~N}_{1}$ Clade 2.3.2.1C virus in migratory birds, 2014-2015. Virol Sin. 2016;31(4):300-5. DOI: 10.1007/S12250-016-3750-4 PMID: 27405930

15. Naguib MM, Kinne J, Chen H, Chan KH, Joseph S, Wong $\mathrm{PC}$, et al. Outbreaks of highly pathogenic avian influenza $\mathrm{H}_{5} \mathrm{~N}_{1}$ clade $2.3 .2 .1 \mathrm{C}$ in hunting falcons and kept wild birds in Dubai implicate intercontinental virus spread. J Gen Virol. 2015;96(11):3212-2. DOI: 10.1099/jgv.0.000274 PMID: 26350163

16. Tassoni L, Fusaro A, Milani A, Lemey P, Awuni JA, Sedor VB, et al. Genetically Different Highly Pathogenic Avian Influenza $\mathrm{A}\left(\mathrm{H}_{5} \mathrm{~N}_{1}\right)$ Viruses in West Africa, 2015. Emerg Infect Dis. 2016;22(12):2132-6. DOI: 10.3201/eid2212.160578 PMID: 27389972

17. Claes F, Morzaria SP, Donis RO. Emergence and dissemination of clade 2.3.4.4 $\mathrm{H}_{5} \mathrm{Nx}$ influenza viruses-how is the Asian HPAl $\mathrm{H}_{5}$ lineage maintained.Curr Opin Virol. 2016;16:158-63. DOI: 10.1016/j.coviro.2016.02.005 PMID: 26991931

18. Lee DH, Torchetti MK, Winker K, Ip HS, Song CS, Swayne DE. Intercontinental Spread of Asian-Origin $\mathrm{H}_{5} \mathrm{~N} 8$ to North America through Beringia by Migratory Birds.J Virol. 2015;89(12):65214. DOI: $10.1128 /$ JVI.00728-15 PMID: 25855748

19. ProMED-mail. Avian influenza $\mathrm{H}_{5} \mathrm{~N} 8$ Germany. Archive. 2016;4641281(: 20161119):19. Available from: http://www. promedmail.org/post/20161119.4641281

20. ProMED-mail. Avian influenza $\mathrm{H}_{5} \mathrm{~N} 8$ Israel. Archive. 2016;4628359(: 20161114):14. Available from: http://www. promedmail.org/post/4628359

21. Munster VJ, Baas C, Lexmond P, Waldenström J, Wallensten A, Fransson T, et al. Spatial, temporal, and species variation in prevalence of influenza $A$ viruses in wild migratory birds. PLoS Pathog. 2007;3(5):e61. DOI: 10.1371/journal.ppat.0030061 PMID: 17500589

22. Peiris JS, Cowling BJ, Wu JT, Feng L, Guan Y, Yu H, et al. Interventions to reduce zoonotic and pandemic risks from avian influenza in Asia. Lancet Infect Dis. 2016;16(2):252-8. DOI: 10.1016/S1473-3099(15)00502-2 PMID: 26654122

23. Sims LD. Intervention strategies to reduce the risk of zoonotic infection with avian influenza viruses: scientific basis, challenges and knowledge gaps.Influenza Other Respi Viruses. 2013;7(Suppl 2):15-25. DOI: 10.1111/irv.12076 PMID: 24034479

24. Höper D, Hoffmann B, Beer M. Simple, sensitive, and swift sequencing of complete $\mathrm{H}_{5} \mathrm{~N}_{1}$ avian influenza virus genomes. Clin Microbiol. 2009;47(3):674-9. DOI: 10.1128/JCM.01028-08 PMID: 19109473

25. European Commission (EC). 2006/437/EC: Commission Decision of 4 August 2006 approving a Diagnostic Manual for avian influenza as provided for in Council Directive 2005/94/EC (notified under document number C(2006) 3477) (Text with EEA relevance). OJ L 237, 31.8.2006, p. 1-27. Brussels: EC; 2006. Available from: http://eur-lex.europa.eu/legal-content/EN/ TXT/?uri=CELEX\%3A32006D0437

26. World Organisation for Animal Health/Food and Agriculture Organization of the United Nations (OIE/FAO). Network of expertise on animal influenza. Influenza A Cleavage sites. Paris/Rome: OIE/FAO; 2015. [Accessed 01 Dec 2016] Available from: http://www.offlu.net/fileadmin/home/en/resourcecentre/pdf/Influenza_A_Cleavage_Sites.pdf

27. Influenza research database (IRD). [Internet]. Highly Pathogenic $\mathrm{H}_{5} \mathrm{~N}_{1}$ Clade Classification Tool. [Accessed 21 Nov 2016]. Available from: https://www.fludb.org/brc/ h5n1Classifier.spg?method=ShowCleanInputPage\&decorator= influenza

28. Hoffmann B, Harder T, Lange E, Kalthoff D, Reimann I, Grund $C$, et al. New real-time reverse transcriptase polymerase chain reactions facilitate detection and differentiation of novel $\mathrm{A} / \mathrm{H}_{1} \mathrm{~N}_{1}$ influenza virus in porcine and human samples. Berl Munch Tierarztl Wochenschr. 2010;123(7-8):286-92.PMID: 20690540

29. Spackman E, Senne DA, Bulaga LL, Myers TJ, Perdue ML, Garber LP, et al. Development of real-time RT-PCR for the detection of avian influenza virus. Avian Dis. 2003;47(3) Suppl;1079-82. DOI: 10.1637/0005-2086-47.S3.1079 PMID: 14575115

30. Hoffmann B, Hoffmann D, Henritzi D, Beer M, Harder TC. Riems influenza a typing array (RITA): An RT-qPCR-based low density array for subtyping avian and mammalian influenza a viruses.Sci Rep. 2016;6:27211. DOI: 10.1038/srep27211 PMID: 27256976

31. Hoffmann B, Harder T, Starick E, Depner K, Werner O, Beer M. Rapid and highly sensitive pathotyping of avian influenza $A$ $\mathrm{H}_{5} \mathrm{~N}_{1}$ virus by using real-time reverse transcription-PCR.J Clin Microbiol. 2007;45(2):600-3. DOI: 10.1128/JCM.01681-06 PMID: 17182758

32. Ampofo WK, Azziz-Baumgartner E, Bashir U, Cox NJ, Fasce R, Giovanni M, et al. , WHO Writing Group. Strengthening the influenza vaccine virus selection and development process: Report of the 3rd WHO Informal Consultation for Improving Influenza Vaccine Virus Selection held at WHO headquarters, Geneva, Switzerland, 1-3 April 2014.Vaccine. 2015;33(36):4368-82. DOI: 10.1016/j.vaccine.2015.06.090 PMID: 26148877

33. Wong S-S, Webby RJ. Traditional and new influenza vaccines. Clin Microbiol Rev. 2013;26(3):476-92. DOI: 10.1128 / CMR.00097-12 PMID: 23824369

34. European Centre for Disease Prevention and Control (ECDC). Outbreak of highly pathogenic avian influenza. $\mathrm{A}\left(\mathrm{H}_{5} \mathrm{~N} 8\right)$ in Europe - 18 November 2016. Stockholm: ECDC; 2016. [Accessed 21 Nov 2016] Available from: http://ecdc.europa.eu/ en/publications/Publications/risk-assessment-avian-influenzaH5N8-europe.pdf

35. Kis Z, Jones J, Creanga A, Ferdinand K, Inui K, Gerloff N, et al. Real-time RT-PCR assay to differentiate clades of $\mathrm{H}_{5} \mathrm{~N}_{1}$ avian influenza viruses circulating in Vietnam. J Virol Methods. 2013;193(2):452-8. DOI: 10.1016/j.jviromet.2013.06.023 PMID: 23850699

36. Abdelwhab SM, Erfan AM, Grund C, Ziller M, Arafa A-S, Beer M, et al. Simultaneous detection and differentiation by multiplex real time RT-PCR of highly pathogenic avian influenza subtype $\mathrm{H}_{5} \mathrm{~N}_{1}$ classic (clade 2.2.1 proper) and escape mutant (clade 2.2.1 variant) lineages in Egypt. Virol J. 2010;7(1):260. DOI: 10.1186/1743-422X-7-260 PMID: 20929539

\section{License and copyright}

This is an open-access article distributed under the terms of the Creative Commons Attribution (CC BY 4.0) Licence. You may share and adapt the material, but must give appropriate credit to the source, provide a link to the licence, and indicate if changes were made.

This article is copyright of the authors, 2017. 